\title{
Effect of the Triplet State on the Random Telegraph Signal in Si n-MOSFETs
}

\author{
Enrico Prati and Marco Fanciull:* \\ Laboratorio Nazionale Materiali e Dispositivi per la Microelettronica, \\ Consiglio Nazionale delle Ricerche - Istituto Nazionale per la \\ Fisica della Materia, Via Olivetti 2, I-20041 Agrate Brianza, Italy \\ Giorgio Ferrari and Marco Sampietro \\ Dipartimento di elettronica e informazione, Politecnico di Milano, \\ P.za Leonardo da Vinci 32, I-20133 Milano, Italy
}

\begin{abstract}
We report on the static magnetic field dependence of the random telegraph signal (RTS) in a submicrometer silicon n-metal-oxide-semiconductor field-effect transistor. Using intense magnetic fields and ${ }^{3} \mathrm{He}$ temperatures, we find that the characteristic time ratio changes by 3 orders of magnitude when the field increases from 0 to $12 \mathrm{~T}$. Similar behaviour is found when the static field is either in-plane or perpendicular to the two dimensional electron gas. The experimental data can be explained by considering a model which includes the triplet state of the trapping center and the polarization of the channel electron gas.
\end{abstract}

Random telegraph signal (RTS), consisting of the random switching of the current in a metal-oxidesemiconductor field effect transistor between two levels, has been studied for decades [1, 2, 3]. The quantum theory of the RTS in silicon metal-oxide-semiconductor field-effect transistors (MOSFETs) is based on inelastic capture and emission of charge by a trapping center near the $\mathrm{Si} / \mathrm{SiO}_{2}$ interface. Each tunnel transition of the electron between the trap and the channel is assisted by multiphonon emission and absorption, and reflects the effect of electrostatic Coulomb barriers of the charges present in the device [4].

Recently RTS has been proposed as a viable way towards single spin detection, a key issue towards solid state quantum information processing (QIP) [5, 6]. In particular, for those QIP schemes in which the electron spins are the qu-bits, read-out of the spin state can be achieved by monitoring the variation of the RTS in electron spin resonance conditions. In this scheme the MOSFET is operated in a static magnetic field and under microwave irradiation [7, 8, 9]. Hence, the response of a trap at the $\mathrm{Si} / \mathrm{SiO}_{2}$ interface of a MOSFET placed in a static magnetic field and the occupation of the trap spin states are of considerable interest.

The effect of the static magnetic field on the RTS has been recently described [10], and a model has been proposed to take into account the characteristic time ratio $r=\frac{\tau_{\text {high }}}{\tau_{\text {low }}}$ change as a function of the magnetic field, where $\tau_{\text {high }}$ and $\tau_{\text {low }}$ are the average characteristic times of the two current levels. However, at very low temperatures and high magnetic fields, significant discrepancies between the experimental data obtained and the model have been observed.

We report on the investigation of the RTS parameters as a function of static magnetic fields up to $12 \mathrm{~T}$, both inplane and perpendicular to the two dimensional electron

*Electronic address: marco.fanciulli@mdm.infm.it gas (2DEG) localised in the inversion layer of a $\mathrm{Si}$ MOSFET. Our experiments are performed at liquid ${ }^{3} \mathrm{He}$ temperature where the effects due to the static magnetic field are expected and proved to be strong 10. We also suggest a model which, taking into account the triplet state of the trap, can explain the high field effects.

The investigated devices are commercial n-channel MOSFETs realised on a p-well, with a length of $0.18 \mu \mathrm{m}$ and a width of $0.28 \mu \mathrm{m}, 3.5 \mathrm{~nm}$ thick gate oxide, and a threshold voltage at low temperature $V_{\mathrm{th}}=536 \mathrm{mV}$. The current $I_{D}$ flowing through drain and source is measured by a transimpedance amplifier whose output is sampled and digitized for off-line processing. The bandwidth of the amplifier extends from $0.1 \mathrm{~Hz}$ to about $160 \mathrm{kHz}$. The transistor and the electronic are powered by independent batteries to avoid power-line pick-ups and interferences. Extreme caution has been devoted to the suppression of all the spurious signals due to the instruments. The setup allowed us to characterize traps with characteristic times down to few $\mu$ s. Each acquisition was taken in a time interval between 60 and $150 \mathrm{~s}$. The samples were mounted on a gold plated dual-in-line made with high thermal conductivity ceramic placed in thermal contact with a copper coldfinger. All the contacts to the source, drain, and gate were directly accessible through bonding pads and connected to the dual in line pads. The coldfinger was cooled at ${ }^{3} \mathrm{He}$ cryogenic temperature in vacuum at the nominal value of $T_{c f}=245 \mathrm{mK}$. The cryostat was operated into a $12 \mathrm{~T}$ Oxford Instruments magnet. The temperature $\Theta$ of the electron gas was slightly higher than the base temperature $T_{c f}$ because of the power dissipation of the current $I_{D}$, observed as a broadening of the single state resonant tunneling conductance peaks versus the gate voltage $V_{G}$ 11.

Suitable traps for the RTS detection were identified. Each sample revealed traps at several gate voltages above the threshold and activated in a voltage range $\Delta V_{G} \simeq$ $0.5-2 \mathrm{mV}$. Characteristic times of the traps ranged from tens of $\mu$ s to few ms.

Figure 1 shows the characteristic time ratio $r$ for a 
trap observed with $V_{G}=560.7 \mathrm{mV}$ and $V_{D}=15.7 \mathrm{mV}$ as a function of an external static magnetic field $B \|$ in the plane of the channel and oriented in the direction perpendicular to the electron current flow (inset of Fig. 1). The drain current was $I_{D}=37.3 \mathrm{nA}$. The current state low of the RTS corresponds to the physical state of an electron captured by the trap, because the ratio $r$ decreases by increasing $V_{G}$ (therefore lowering the trap energy $\left.E_{T}\right)$.

In order to determine whether the trap was singly occupied, and therefore paramagnetic, and capable of capturing a second electron (type $1 \rightarrow 2$ ) or an empty trap capable of capturing an electron $(0 \rightarrow 1)$, we have analysed the dependence of $r$ on the magnetic field B [9]. For a singly occupied trap, the highest energy level of the Zeeman doublet increases as the magnetic field increases. We observed that the trap under investigation increases its permanence in the current state high by increasing the magnetic field, so that the highest energy level becomes on the average less filled, implying that the trap is a $1 \rightarrow 2$ type. Such experimental fact is consistent with the most probable nature of the trap being a $P_{b 0}$ or $P_{b 1}$ at the $\mathrm{Si} / \mathrm{SiO}_{2}$ interface or an $E^{\prime}$ center close to it. The ratio $r$ increases by three orders of magnitude when the magnetic field increases from 0 to $12 \mathrm{~T}$. Such behaviour is qualitatively similar to that observed by Xiao et al.[10]. However, quantitatively, the effect we detect is two orders of magnitude larger.

The same sample was examinated also with the external static magnetic field $B \perp$ perpendicular to the channel. Figure 2 shows the ratio $r$ for another trap observed with $V_{G}=673.8 \mathrm{mV}$ and $V_{D}=1.6 \mathrm{mV}$. At this gate voltage, the channel electron density is $n_{s} \simeq 8 \times 10^{11} \mathrm{~cm}^{-2}$. The current was $I_{D}=35 \mathrm{nA}$. This trap has also been investigated with $B \|$ as discussed later. By applying the same procedure than in the previous case, we found that the active trap involves a $1 \rightarrow 2$ process. Also in this case $r$ increases by more than a factor 10 when the magnetic field $B \perp$ was increased from 0 to $11 \mathrm{~T}$ (Figure 2). A peculiar signature of the measurement is that the RTS disappears when $B \perp$ is in the range $7.75 \mathrm{~T}$ and $9.4 \mathrm{~T}$. $\Delta I$ is defined as the difference between the high and low state current and it vanishes in such field range (Fig. 3). The magneto-induced localization of electrons occurring into the 2DEG at such high magnetic fields can be responsible of such RTS suppression. At low temperature, when the 2DEG is orthogonal to the magnetic field B, the energy levels of the electron gas are discrete (Landau levels) and we point out that the filling factor $\nu=4$ level is at $8.3 \mathrm{~T}$. The Ramo's theorem states that $\Delta I=q v E_{x}$ where $q$ is the electron charge, $v$ the charge velocity component in the direction of the electric field, and $E_{x}$ the applied electric field. We speculate that such vanishing of $\Delta I$ reflects the average value $v=0$ due to localization.

To explain the dependence of $r$ on the magnetic field, we propose a model based on elementary statistical physics that includes the triplet state of the trap and the Zeeman splitting of the channel electrons in addition to the Zeeman splitting of the trap. The model relies on the following assumptions. The effect of the magnetic field is to split the spin degenerate levels of conduction electrons (having g-factor $g_{e}$ [12]) and of the trap (having g-factor $g_{T}$ ) by $E_{Z}^{c}=g_{e} \mu_{B} B$ and $E_{Z}^{T}=g_{T} \mu_{B} B$ respectively, where $\mu_{B}=\frac{e \hbar}{2 m_{e} c}$. Assuming that at high field a two-particle triplet state can be formed, we call $\Delta U$ the difference between the energy in the singlet $S=0$ and in the triplet states $S=1$ of the trap at $B=0$. $\Delta U$ includes both the exchange term and the difference of the Coulomb repulsion between the electrons in the single and triplet states. As suggested by Xiao et al.[10] the Coulomb respulsion can be considered as B field independent. We assume that the inelastic tunnelling of the channel electrons into the trap occurs in two steps: first, the preparation of the electron to the capture process because of both the interaction with the potential of the trapping center and the multiphonon emission due to the relaxation of the lattice near the defect [13]; second the capture itself by tunneling (and viceversa for the emission) [4]. The temperature $\bar{\Theta}$ associated to such a process is locally defined and refers to the inelastically scattered electrons prepared to be captured and the paramagnetic electron of the trap. The expression of the ratio $r$ is:

$$
r=\frac{2 e^{\beta\left(E_{T}+U_{S}-E_{F}\right)}\left(\cosh \beta\left\langle E_{Z}\right\rangle+\cosh \beta \frac{\delta}{2}\right)}{1+e^{-\beta \Delta U}\left(1+\cosh \beta E_{Z}^{T}\right)}(1)
$$

where $E_{T}$ is the energy level of the empty trap, $U_{S}$ is the Coulomb energy of the doubly occupied trap, $\beta=k \bar{\Theta}$, $k$ is the Boltzmann constant, $\left\langle E_{Z}\right\rangle=\frac{E_{Z}^{T}+E_{Z}^{C}}{2}$ is the average value of the two Zeeman splittings of the channel and of the trap respectively, and $\delta=\left|E_{Z}^{T}-E_{Z}^{C}\right|$. Since the g-factors of the free electron and of the defect are very close (assuming that the trap is a $P_{b 0}, P_{b 1}$ or an $E^{\prime}$ center), then $\cosh \beta \frac{\delta}{2} \approx 1$. The denominator differs from unity because of a term which depends on the inclusion of the triplet state. At high B field such term dominates and suppresses the exponential behaviour of $r$ as a function of B. Eq. (1) has been used to fit the experimental data when the B field was either parallel (Figure 1) or perpendicular (Figure 2) to the channel. The fit was performed by considering the logarithm of the Eq. 1 to fit the logarithm of the experimental data so all the points were weigthed in the same way. The three parameters used for the model which includes the triplet state were the local temperature $\bar{\Theta}$ of the electrons interacting with the trap site, a multiplicative constant, and $\Delta U$, while only the first two parameters were used if only the $S=0$ state was considered. For both the experiments $(B \|$ and $B \perp$ to the $2 \mathrm{DEG}$ ) a significantly better fit was obtained by including the triplet state. The improvement of the fit including the polarization of the 2DEG was less important, but not negligible. In the $B \|$ and $B \perp$ cases, the fit including the triplet state gave a local temperature $\bar{\Theta}$ of $1.5 \mathrm{~K}$ and $2.3 \mathrm{~K}$ respectively, with an error of about $5 \%$. The second trap has been measured also applying $B \|$ in the same operating conditions. $B \|$ was 
swept to an induction of $12 \mathrm{~T}$ in both the positive and negative directions. The fit gave the same temperature obtained for the $B \perp$ case. The triplet state model gives a $\Delta U \approx 5 k_{B} \bar{\Theta}$ where $\bar{\Theta}$ is the temperature calculated by the same fit for both traps. At first sight it could be surprising that a higher temperature is found in the second trap (B field perpendicular to the $2 \mathrm{DEG}$ ) where a smaller drain voltage was applied and a smaller current was flowing through the channel. However, this fact is compatible with the scheme proposed, because the local electron temperature in the neighborhood of the defect depends not only on the power dissipated by the current, but also on the trap distance from the $\mathrm{Si} / \mathrm{SiO}_{2}$ interface, its proximity to the source along the channel, and its energy level, via the number of phonons involved in the inelastic tunneling process.

To conclude, we have demonstrated experimentally that the ratio between the characteristic times of the RTS in a silicon n-MOSFET may change by three orders of magnitude in presence of an intense magnetic field up to $12 \mathrm{~T}$ parallel to the 2DEG. The same behaviour, although less pronounced, was observed also in another trap by applying a perpendicular field. To explain quantitatively the change of the ratio $r$ as function of $\mathrm{B}$, and the suppression of the exponential behavior when high magnetic fields are reached, we propose a model that includes the involvement of the triplet state of the trap and the polarization of the $2 \mathrm{DEG}$.

\section{Acknowledgments}

The authors would like to thank Ivar Martin (Los Alamos), Dima Mozyrsky (Clarkson University, NY), and Hong Wen Jiang (UCLA, CA), for the useful discussion, Vittorio Pellegrini, Vincenzo Piazza, Pasqualantonio Pingue for the cryomagnet time and the support at the Laboratorio Polvani of NEST CNR-INFM and SNS, Pisa, Italy.
[1] K. S. Ralls et al., Phys. Rev. Lett. 52, 228 (1984)

[2] K. Kandiah, M.O. Deighton, and F.B. Whiting, J. Appl. Phys., 66, 2, 937 (1989)

[3] A. Longoni, E. Gatti, and R. Sacco, J. Appl. Phys., 78, 10, 6283 (1995)

[4] A. Palma, A. Godoy, J.A. Jimenez-Tejada, J.E. Carceller, J.A. Lopez-Villanueva, Phys. Rev. B, 56, 15, 9565 (1997)

[5] B. E. Kane, Nature (London) 393, 133 (1998)

[6] D. Loss and D. P. DiVincenzo, Phys. Rev. A 57, 120 (1998)

[7] R. Vrijen et al., Phys. Rev. A 62, 12306 (2000)

[8] D. Mozyrsky, V. Privman, and M. L. Glasser, Phys. Rev. Lett. 86, $5112(2001)$

[9] M. Xiao, I. Martin, E. Yablonovitch, and H. W. Jiang, Nature, 430, 435 (2004)

[10] M. Xiao, I. Martin, and H. W. Jiang, Phys. Rev. Lett. 91, 078301 (2003)

[11] A.D. Stone and P.A. Lee, Phys. Rev. Lett. 54, 11, 1196 (1985)

[12] J. Wallace and R. H. Silsbee, Phys. Rev. B 44, 12964 (1991)

[13] C. H. Henry and D. V. Lang, Phys. Rev. B 15, 2, 9891016 (1977)

\section{FIGURE CAPTIONS}

Fig.1 The ratio $r$ as a function of $B \|$. Because of the power dissipated by the current, the temperature of the electrons involved in the RTS of the trap here considered has been evaluated being $1.5 \mathrm{~K}$, instead of the nominal coldfinger temperature of $245 \mathrm{mK}$, by fitting the experimental results (black points) with the Eq. 1 (line). Inset: the magnetic field is in the plane of the 2DEG and perpendicular to the current channel.

Fig.2 The ratio $r$ as a function of $B \perp$. The experimental measurement (black points) has been fitted by using Eq. 1, obtaining a local temperature $2.3 \mathrm{~K}$, instead of the nominal coldfinger temperature of $245 \mathrm{mK}$. The RTS disappears between $7.75 \mathrm{~T}$ and $9.4 \mathrm{~T}$. The inset shows the direction of the magnetic field, orthogonal to the sample.

Fig.3 The $\Delta I$ changes as a function of the magnetic field $B \perp . \Delta I$ vanishes between $7.75 \mathrm{~T}$ and 9.4 T. This fact should be related to magnetoinduced localization of conduction electrons. At the electron density of the 2DEG used during the measurement, the Landau level $\nu=4$ is at $8.3 \mathrm{~T}$.

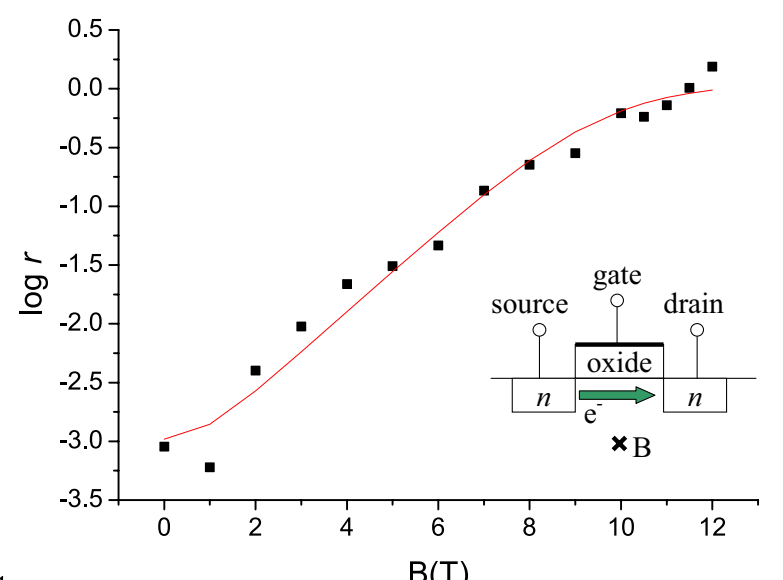

Figure 1 


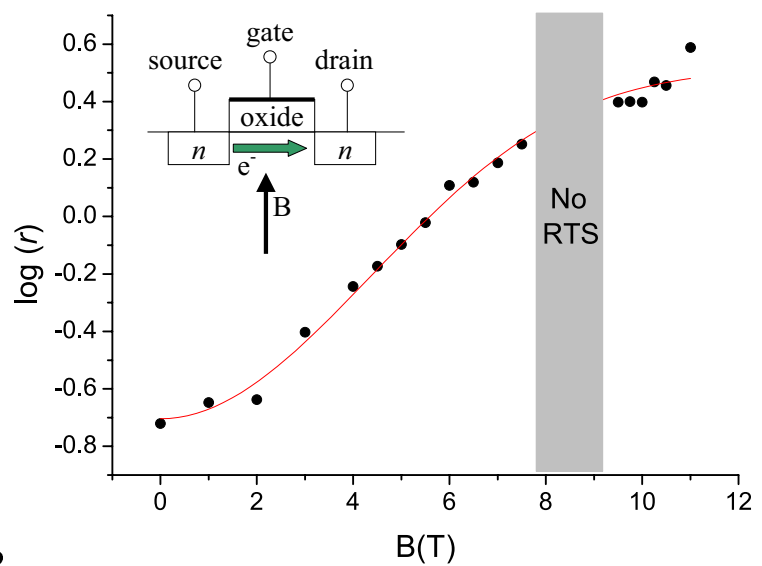

Figure 2

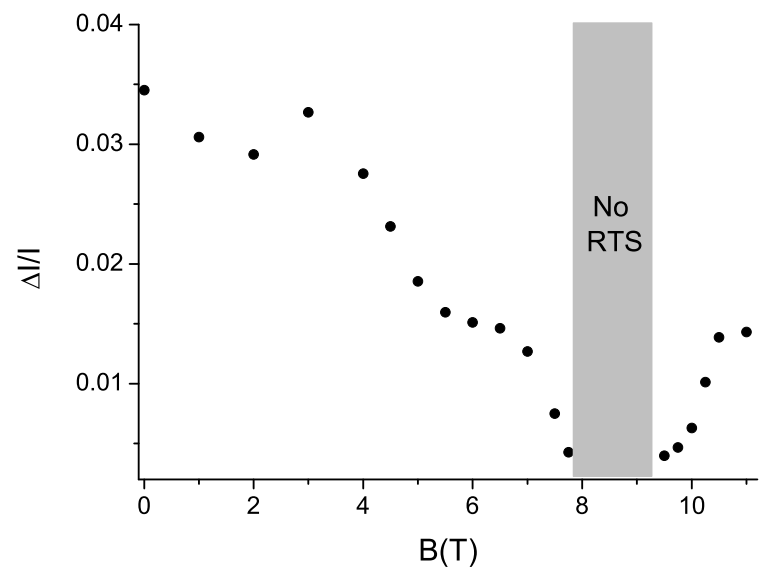

Figure 3 